Rozalia Sasor (1)

Uniwersytet Jagielloński, Kraków rozalia.kosmider-sasor@uj.edu.pl

\title{
El concepto de la guerra justa en el Dotzè de Francesc Eiximenis
}

\begin{abstract}
Resumen:
El artículo presenta el concepto de la guerra justa elaborado por Francesc Eiximenis (1327/32-1409), escritor y fraile franciscano, uno de los autores medievales más importantes en lengua catalana, expuesto en el duodécimo libro (el Dotzè) de la gran enciclopedia cristiana Lo Crestià, dedicado a las cuestiones político-sociales.
\end{abstract}

Palabras clave: Francesc Eiximenis, guerra justa, Lo Crestià, Dotzè

\begin{abstract}
:
The Concept of the Just War in Dotzè by Francesc Eiximenis

The article presents the concept of the just war elaborated by Francesc Eiximenis (1327/32-1409), a writer and Franciscan friar, and one of the most important medieval authors of the Catalan language, which was exposed in the twelfth book (the Dotzè) of the Christian encyclopaedia Lo Crestià, dedicated to political-social issues.
\end{abstract}

Key words: Francesc Eiximenis, just war, Lo Crestià, Dotzè 


\section{Francesc Eiximenis}

Francesc Eiximenis, fraile franciscano y autor polifacético y enciclopedista, nació entre los años 1327 y 1332, en Gerona, según la introducción al Primer del Crestià donde leemos: "l'actor de aquest libre és stat, aprés Déu, un religiós de l'orde dels frares menors, apellat frare Francesc Eiximenis, natural de la ciutat de Girona" (Riquer, 1984: 314). ${ }^{1}$ También confirma su procedencia catalana el fraile Luke Wadding, el cronista más reconocido de la Orden Franciscana, que en su historia de los frailes menores Annales Minorum apunta: "Franciscus Ximenius, alter Gerundensis, Catalanus, non Valentinus", subrayando que su presunto origen valenciano no está fundado en los documentos de la época (Hauf, 1990: 60). Eiximenis, muy pronto, a la edad de unos 20 años, ingresó en la Orden de su ciudad natal y fue ordenado sacerdote en Barcelona unos cinco años después. Para completar su formación, el fraile Francesc emprendió diferentes viajes por toda la Europa cristiana, visitando los más importantes centros culturales de su tiempo, como París, Colonia u Oxford, así como las regiones vecinas de la Corona de Aragón - Italia (Roma y, sobre todo, el monte Alverna - un importante centro de la espiritualidad franciscana) y Provenza. En 1365 Eiximenis pasó algún tiempo en la sede pontificia de Aviñón durante el pontificado de Urbano V; en los años setenta lo encontramos relacionado con el municipio de Barcelona y con la corte del rey Pedro III (IV) el Ceremonioso donde desempeña funciones diplomáticas. Sus dotes, apreciadas no solo por el mismo rey, sino también por los hijos de este: el futuro Juan I el Cazador y Martín el Humano, le permitieron obtener el grado de Maestro en Teología el 25 de marzo de 1374 en el studium de Tolosa. En 1383 Eiximenis fue enviado a Valencia, donde su renombre de gran docto y escritor le propició un éxito inmediato. Residió en esta ciudad hasta el año

${ }^{1}$ La breve biografía de Francesc Eiximenis presentada aquí se basa en tres fuentes primordiales: D. Guixeras, S. Martí (2014), "Eiximenis i la prosa doctrinal del segle XIV", en: Història de la literatura catalana de A. Broch, la monografía de A. Hauf (1990), D'Eiximenis a sor Isabel de Villena y la fundamental Història de la literatura catalana de M. de Riquer (1984). 
1408 dedicando su tiempo a la actividad política, a la escritura y a la predicación. Durante el último año de su estancia en Valencia fue llamado por el Papa Luna al concilio de Perpiñán -ciudad perteneciente por aquel entonces a la Corona de Aragón- y se mudó por última vez. Consagrado por el antipapa Patriarca de Jerusalén y obispo de Elna, tal vez como remuneración por su fidelidad, permaneció en la sede pontificia hasta 1409, cuando murió.

\section{El Dotzè llibre del Crestià}

A pesar de la intensa actividad política -sobre todo en Valencia, en la que actuó como consejero de los Jurats, por petición de quienes escribió su conocido tratado de cómo gobernar una ciudad, Regiment de prínceps e de comunitats,- Francesc Eiximenis fue un autor prolífico que escribía tanto en latín como en catalán. Entre las obras escritas en lengua vulgar, Lo Crestià fue la más ambiciosa. Redactada entre los años 1379 y 1387, fue pensada como una gran enciclopedia cristiana dirigida a toda la gente sencilla para servirle de guía de buena vida en la fe. Era un proyecto monumental: tenía que integrar trece volúmenes, de los que Eiximenis logró terminar tan solo cuatro; pero, por fortuna, se ha conservado el plano general de la suma de Eiximenis que nos permite captar el programa doctrinal previsto para la obra. No obstante, los cuatro volúmenes conservados del Crestià, así como los que nos han sido legados como proyecto, se podrían organizar en tres partes temáticas principales, en las que la primera era una introducción general a la fe cristiana entendida como la única religión verdadera y correspondía al libro Primer; la segunda (libros Segon y Terç) explicaba cómo el buen cristiano debería enfrentarse a las tentaciones, los vicios y pecados a los que la naturaleza humana está inclinada como consecuencia de haber cometido el pecado original. Los restantes diez volúmenes, de los que Eiximenis logró escribir solamente el Dotzè dedicado a la gobernación de la comunidad, tenían que exponer cómo gracias a la intervención divina se podría aplicar la doctrina cristiana a la vida y a todas las actividades humanas (Guixeras, Martí, 2014: 36). A su vez, el libro Dotzè fue concebido por su autor como la suma 
de dos partes diferentes de las que la primera (tratados I-II-III-IV) desarrollaba los temas relacionados con el gobernar de la comunidad, explicados en el Regiment de prínceps e de comunitats, y la segunda (tratados V-VI-VII-VIII) correspondía más bien al género de speculum principis, ya que describía con considerable precisión todos los aspectos del oficio real: desde cómo ejecutar el poder real hasta las referencias a la vida privada del monarca (Eiximenis, 1986: XIV).

El preámbulo nos ofrece también una información sobre los criterios que el autor seguía a la hora de escribir y que, en efecto, han formado su estilo. Eiximenis confiesa que ha decidido hablar de manera "simple i grosera" (Eiximenis, 1983: 37), ${ }^{2}$ sin "curioses al legacions ne tocacions d'autoritats, e d'originals ne d'altres dits científics", y sin "al·legar res en llatí, sinó fort poc" para no impedir la comprensión del texto. Por eso los extractos en latín, aunque sean de párrafos bíblicos generalmente conocidos, van acompañados de las traducciones al catalán. Así mismo, las citas de la Escritura o de las autoridades tienen muy a menudo una forma simplificada y adolecen de la habitual precisión rigurosa de la escolástica, porque su finalidad es transmitir el concepto de manera "pus informativa e pus profitosa" posible y no "segons que jau la letra, de paraula a paraula o de punt a punt". Eiximenis repite con frecuencia que no quiere entrar en las sutilezas teológicas por ser estas demasiado difíciles para un hombre lego; de este modo su manera de proceder está entre un compilador y un comentador que desarrolla y amplía el texto original con su propio razonamiento, anécdotas y proverbios para aclarar los párrafos más complicados. Sin embargo, la afirmación eiximeniana de no escribir para los letrados, no la deberíamos tomar al pie de la letra, ya que varias partes de su obra alcanzan un alto nivel estilístico de prosa doctrinal (Hauf, 1983: 18).

El texto del Crestià tiene una estructura plenamente escolástica, con un razonamiento dividido en partes, ordenado, y una argumentación minuciosa, amplia, frecuentemente reiterativa. Pero al contrario de otras obras de este tipo, la prosa de Eiximenis intercala observaciones

${ }^{2}$ Todas las citas del preámbulo al Primer del Crestià provienen de la edición de A. Hauf (1983: 37). 
marginales de carácter costumbrista, histórico y didáctico, ya que el estilo del tratado está determinado por la voluntad divulgativa del autor. Si bien un lector contemporáneo puede hallar el lenguaje de Eiximenis demasiado redundante o difuso, sus coetáneos lo veían de manera muy diferente. El estilo del autor franciscano, marcado por una abundancia de anécdotas, vivas y pintorescas, atraía el interés de sus lectores que guiados por un texto atractivo y sugerente, divertido e incluso satírico, asimilaban la lección moral. La gran obra de Eiximenis no puede ser considerada un tratado genial ni tampoco muy original, como no lo puede ser una enciclopedia, pero sí que es un escrito excepcional gracias al talante personal que le ha dado su autor, gran erudito y observador agudo de la vida cotidiana, que ofreció en Lo Crestià un panorama amplio de la sociedad catalana de finales del siglo XIV, imprescindible para conocer la Edad Media.

\section{El concepto eiximeniano de la guerra justa}

La teoría de la guerra justa, que a su vez formó el principio fundador de la ideología de la caballería cristiana, consiste en un análisis de cuándo y en qué condiciones una comunidad puede usar la fuerza contra otra. El origen de ius bellis remite a la práctica y filosofía de los antiguos, primeramente de los israelitas y después de los griegos y romanos, como Aristóteles o Cicerón. Sin embargo, de entre los autores que comentaron el tema, los que más influyeron en los escritos militares de Eiximenis fueron san Agustín, Juan de Salisbury, el papa Inocencio III y Enrico de Susa. En el mundo cristiano el concepto de la guerra justa aparece por primera vez en la obra De civitate Dei de Agustín de Hipona, incorporado al pensamiento sobre la gobernación justa que debía basarse en la defensa y el mantenimiento del orden social y de la paz. El Doctor de la Gracia expone su doctrina de manera concisa, lo que facilita divisar los rasgos más importantes de su teoría política, que son: justicia, orden y paz, las mismas que forman las raíces del pensamiento eiximeniano. En cambio, el Policraticus de Juan de Salisbury, el tratado político más influyente de su época sobre el arte de bien gobernar, presenta la doctrina militar como una parte de 
la gran metáfora de la comunidad vista como un cuerpo, dirigido por la cabeza - el príncipe, y defendida por dos manos: la derecha que es el ejército y la izquierda, los funcionarios. Sin embargo, Juan de Salisbury, a pesar de exponer de manera detallada la singularidad del oficio de los militares, comparados con el estado eclesiástico, y la importancia de varios aspectos prácticos enlazados con el servicio guerrero, no reflexiona sobre las definiciones de tales conceptos básicos como el de la paz y de la guerra, ni tampoco propone alguna visión teórica de la guerra justa (Goltz, 2016: 62). Eiximenis establece la base legal del concepto de la guerra justa en los vastos escritos jurídicos de dos autores expertos en derecho canónico, el papa Inocencio III y Enrico de Susa, llamado Hostiensis, repetidamente citados y tratados como máxima autoridad en cuestiones de orden legal de campañas militares.

Aparte de los tratados de influencia ideológica en los escritos eiximenianos, hay que mencionar otro tipo de textos que le servían al autor franciscano de inagotable fuente de ejemplos y anécdotas que ilustraban el razonamiento teórico. Aquí, el papel fundamental lo desempeña la Biblia, y sobre todo el Antiguo Testamento, al que Eiximenis se refiere asiduamente y, de entre los autores paganos, Valerio Máximo, una gran autoridad en cuanto a los temas militares en la Edad Media. Desgraciadamente, es imposible revelar hoy en día todas las fuentes de erudición eiximeniana, porque los relatos de carácter explicativo no siempre van acompañadas de referencias bibliográficas y pueden derivarse tanto de obras concretas todavía no identificadas como de los epítomes, tan abundantes en la época medieval. En este campo, la gran obra de Eiximenis deja a los investigadores un amplio terreno virgen por explorar. ${ }^{3}$ Sin embargo, los defectos bibliográficos no impiden por completo exponer el pensamiento del fraile Francesc, que, a su vez, era una fuente para otros escritores, como por ejemplo Joanot Martorell, que fundaron su concepto de la guerra justa en las ideas elaboradas en el Dotzè.

${ }^{3}$ Para una aproximación a las fuentes eiximenianas cf. la introducción de Hauf, A. (2009) al Regiment de la cosa pública, pp. 11-52. 


\section{Ius ad bellum}

\section{Condición primera: la buena intención}

Aunque la primera parte del Dotzè abarca un extenso tratado de caballería y de la guerra medieval (c. 213-337), es en la segunda donde Eiximenis expone su pensamiento en cuanto a la guerra justa (c. 655659) vinculado con los consejos generales al príncipe, dedicados a los temas de la paz y la guerra (c. 651-654), y un extenso comentario sobre la guerra entre los vasallos (c. 660-668). En primer lugar Eiximenis expone la principal condición de ius ad bellum que según su opinión es la buena intención de quien emprende la guerra, que normalmente es el príncipe, y que identifica con el sometimiento a la ley divina. La buena intención es también el factor sine qua non para obtener la victoria, porque, como amonesta el franciscano, Dios otorga el premio y ayuda a derrotar a los enemigos solamente a los que obedecen fielmente sus mandamientos: "Si vosaltres volets servar mos manaments, vençrets vostres enemichs e yo·ls faré caure davant vostres peus" (Eiximenis, 1986: 456). ${ }^{4}$ Para ilustrar su consejo Eiximenis alude a varias historias bíblicas, como la de Holofernes y Aquior quien advierte al comandante de la hueste babilónica de que no intente guerrear contra los israelitas si sabe que no han cometido ningún pecado, porque si infringe dicha regla su Dios los protegerá y Holofernes perderá la batalla (Jdt 5); ${ }^{5}$ o las de los grandes héroes del Antiguo Testamento: Sansón, que con una quijada de asno mató a mil filisteos (Jue 15) o Jonatán, hijo de Saúl, que solo con un ayudante (Eiximenis lo llama 'escudero') hizo huir a las huestes filisteas de diez mil hombres (1 Sam 14). La lista de guerreros invencibles la cierra Judas Macabeo, ganador de todas las batallas gracias a su confianza en "la justícia sua e de la ajuda de nostre senyor Déu” (Eiximenis, 1986: 457), pero que perdió la vida al fiarse de los romanos. Otro ejemplo de la derrota causada por el

${ }^{4}$ En lo sucesivo todas las citas de Eiximenis provienen de la misma edición del año 1986, así que entre paréntesis se indica solamente el número de página de dicha edición donde aparece el fragmento citado.

${ }^{5}$ En este fragmento Eiximenis se refiere al Libro de Judith, pero coloca la cita, erróneamente, en el capítulo sexto en lugar del quinto. 
pecado procede del Libro de Josué que narra la toma de Jericó y el pecado de Acán al tomar para sí de los bienes conquistados que debían ser destruidos. Su acción, contraria a la ley divina, hizo que todos los israelitas fueran culpables ante el Señor y provocó la derrota de las huestes israelíes en el ataque a $\mathrm{Ai}(\operatorname{Jos} 7)$.

La idea de la confianza en Dios respaldada por la pureza moral - un concepto básico del teocentrismo medieval - es el fundamento del pensamiento del autor franciscano acerca de la guerra justa. Según Eiximenis el poder militar proviene de la voluntad divina y Dios, al ser el principio de todo el orden del mundo, representado por el gobierno de los príncipes, castiga con una derrota a los que violan sus leyes. En el capítulo 656 Eiximenis expone este parecer suyo de manera explícita: "la victòria de la batalla deu esperar lo príncep e creure fermament que davall de Déu" (p. 458) y le obliga al príncipe a que entre en guerra preparado espiritualmente, porque no puede vencer a sus enemigos sin encomendarse antes al Creador y rogarle que le dé la victoria. El consejo lo funda en la autoridad bíblica del primer Libro de los Macabeos, ${ }^{6}$ del Éxodo donde se relata la famosa batalla contra los amalecitas en la que Moisés tuvo sus brazos levantados el día entero para otorgar la victoria a los israelitas (Ex 17, 11-13) y en el capítulo del Deuteronomio sobre las leyes de guerra en el cual se dice que Dios luchará contra los enemigos de su pueblo (Dt 20, 3). Los ejemplos de la Escritura van acompañados por una serie de anécdotas históricas: sobre el emperador Teodosio el Grande, quien en la batalla del Frígido venció al usurpador Eugenio y a su comandante Arbogastes gracias a una milagrosa nube enviada por Dios que repelía las flechas arrojadas por sus enemigos; o el bien conocido episodio del encuentro (en 452) del papa León I el Magno con Atila, el rey de los hunos (titulado por Eiximenis 'príncep dels gots'), durante el cual se firmó

${ }^{6}$ Eiximenis solamente menciona el párrafo bíblico (1 Mac 3) sin citar el fragmento apropiado, pero sin duda alguna se refiere a la respuesta que Judas dio a sus soldados asustados por la numerosidad del ejército sirio de Serón: "Es fácil que una gran multitud caiga en poder de unos pocos, pues para Dios lo mismo es dar la victoria con muchos que con pocos. En una batalla, la victoria no depende del número de los soldados, sino de la fuerza que Dios da" (1 Mac 3, 18-19). 
un tratado de paz entre el Imperio Romano y los invasores. Según la leyenda citada por el autor franciscano, Atila cedió a las peticiones del papa intimidado por la presencia de un sacerdote viejo con la espada en la mano quien le amenazó: "Si tu no atorgues a aquest tot ço que·t demana, ab aquest coltell te partiré pell mig!" (p. 459). Con estas palabras Eiximenis resalta la preeminencia de la Iglesia sobre el poder del mundo que ve confirmada por la ceremonia de armar caballeros en la cual el combatiente recibe de "mà de la esgleya" la espada y se obliga a honrar a la Iglesia y a sus servidores, evitar todo tipo de acciones injustas contra ellos y suplicar que recen a Dios por él. Además, para garantizar la protección de Dios, el caudillo debería alejar de sus tropas a los pecadores y hombres villanos, y prepararse para la guerra con sabiduría para no tentar a Dios. En este punto Eiximenis deja de lado la doctrina cristiana y pasa a los consejos más prácticos, pero sin dejar de basarse en los fundamentos de la Biblia y recurriendo al antiguo tópico de la sapientia et fortitudo:

E, no contrastant que lo príncep proceesca així com dit és en la batalla, ço és, esperant sa victòria de Déu, emperò ja per això no deu lleixar que no faça ço qui en sí és, car llavors temptaria a Déu; per què deu curar, segons que damunt és dit, que haja ab si hòmens qui sàpien art de batallar, car saviea venç força, segons que diu l'Escriptura. (p. 459)

A continuación fra Francesc aduce los ejemplos de Alejandro Magno, Escipión el Africano y de Jerjes para subrayar la importancia de entrar en guerra con un ejército - aunque no sea numeroso- liderado por hombres experimentados en el arte de batallar, perspicaces y doctos por sus estudios ('philòsofs') o por su experiencia ('hòmens antics'). Y, siguiendo el texto del Policraticus, recuerda el ingenio de los romanos que conquistaron el mundo gracias a su sabiduría y a la práctica de la abstinencia sexual "esquivant (...) delits carnals qui amolexen lo cor" (p. 459). Sin embargo, si un príncipe quiere ganar la guerra, no es suficiente con que disponga de varios conocimientos. Debería también presentar "dolces maneres e atractives als combatents", "amistat e graciocitat als seus" (p. 459) que, según Valerio Máximo, le aseguren la victoria, porque un capitán cruel y orgulloso nunca dará 
tanto coraje a sus soldados como un capitán afable y cordial que soporte las dificultades de una campaña militar compartiéndolas con otros.

\section{Condición segunda: la causa justa}

La segunda condición que el príncipe debe cumplir si piensa comenzar una guerra es la causa justa ('la entenció dreta'), cuyas limitaciones Eiximenis resume en los capítulos 655 y 658: para evitar el pecado, no se puede emprender la guerra por placer o por venganza, ni tampoco a fin de conquistar tierra o fama, o para rebelarse contra el señor, sino solamente por necesidad y para conseguir justicia y paz. Así pues el príncipe tiene derecho a entrar en batalla solo cuando se vea forzado a dicho acto por los enemigos de la cosa pública, "més volent pau que guerra" (p. 464). La reflexión más detallada sobre la legalidad de la lucha se encuentra en el capítulo 657, titulado "Si guerrejat e batallar és cosa leguda", en el cual Eiximenis alega a la autoridad de san Agustín y opina que la guerra es lícita cuando se emprende para castigar los pecados o una conducta contraria a los mandamientos de Dios: "Com pot dir lo príncep que ell servesqua a Déu ab vera temor, si ell no veda ne ponex les coses qui·s fan contra la ley e manament de Déu?" (p. 461), aunque entre los pecados aquel que más justifica la acción militar es el del comportamiento abusivo contra los pobres, como dice el Salmo de Asaf: "¡Librad a los débiles y pobres, / y defendedlos de los malvados!" (Sal 82, 4). ${ }^{7} \mathrm{Al}$ texto de la Escritura lo complementa el pensamiento jurídico del papa Inocencio III que extiende la obligación de proteger a los necesitados en cada situación en la que alguien pierde sus pertenencias, sea un individuo o una comunidad o, atacado, se vea obligado a defender su tierra. Así la causa justa es: "perseguir o refrenar los mals hòmens e defensar los bons (...) demanar ço qui es tolt, voler satisfacció de ses injúries, perssonals o de la comunitat, ${ }^{8}$

${ }^{7}$ Eiximenis cita el fragmento en latín y después lo parafrasea (Eiximenis, 1986: 461).

${ }^{8}$ La causa de la "satisfacció" se encuentra en una contradicción obvia a la prohibición de emprender la guerra por venganza expresada en el capítulo 655. 
(...) deffensió de la terra ajudant a los flachs e impotents en lur justícia a defendre" (p. 462).

Eiximenis termina su razonamiento enumerando otras doce causas - cada una basada en un ejemplo del Antiguo Testamento- que le sirven de móvil para justificar la necesidad de emprender la guerra y que son las siguientes: blasfemia contra Dios originada por el pecado de idolatría; desobediencia a la ley divina y culto de dioses ajenos; traición de un señor por otro, quebranto de la lealtad entre dos señores; rebelión de vasallos; defensa de un delincuente; ofensa pública hecha en la persona del príncipe; demanda de los bienes pertenecientes legalmente al príncipe; contra los enemigos que atacan; contra los enemigos que se sublevan; contra los que ayudan a los enemigos; para liberar a los amigos de manos del enemigo y la última, tratada ampliamente por Juan de Salisbury, contra los tiranos (p. 462-463).

\section{Condición tercera: la autoridad}

La tercera condición de la guerra justa establece que la guerra puede ser declarada solamente por una autoridad de mayor legitimidad ('auctoritat de major', p. 461), o sea, por el rey (el príncipe) o el papa. Eiximenis fundamenta su razonamiento en los escritos del gran conocedor del derecho canónico, el cardenal de Ostia, Enrico de Susa. Según Hostiensis el poder eclesiástico era superior al secular y de esta manera la autoridad adecuada en caso de una guerra en defensa de la fe era en primer lugar la papal y después la del emperador. Sin embargo, como dice el papa Inocencio III, los eclesiásticos, aunque puedan declarar la guerra legalmente, tienen prohibido participar en la lucha personalmente: "prelat no haja dret de menar batalla, ell emperò la aconseguex per lo dret que ha l'esgleya" (p. 462). Si bien la autoridad personal no es la única para ordenar la lucha, la guerra o la batalla se pueden emprender por la autoridad de derecho en todas las cuestiones judicialmente reguladas por falta del mandamiento explícito de la

${ }^{9}$ La defensa de los débiles es también el tópico del juramento que rinden los escuderos durante la ceremonia de ser armados caballeros. 
potestad superior. Incluso se admiten como justas y legítimas las batallas individuales: las ordenadas por el juez o el señor natural (en caso de que el ofensor no quiera remunerar al ofendido) y las establecidas por el derecho natural de cada hombre a defenderse a sí mismo y a sus posesiones. Con la excepción de si el conflicto personal ocurriera entre un laico y un eclesiástico, el sacerdote no podría entrar en la lucha por el derecho que le vedaba derramar sangre (p. 465).

\section{Ius in bello et ius post bellum}

Las cuestiones del ius in bello y post bellum no ocupan mucho espacio en el tratado eiximeniano y su análisis se relaciona con el contexto de la causa justa. Como la batalla se emprende solamente cuando no hay otra posibilidad de resolver el conflicto, se debe luchar con valentía, pero "fer als presos tota misericordia" (p. 464) para no aumentar la desorganización de la comunidad. Eiximenis explica cuál debería ser la relación entre los presos y sus vencedores en el capítulo 659 siguiendo la interpretación de Hostiensis: si la guerra ha sido comenzada por orden del príncipe, o sea, por la autoridad mayor, los presos son cautivos y pertenecen a quienes los han vencido, así que si intentan huir, pecarán. Así mismo, los bienes propios de los presos (excepto los bienes feudales recibidos del señor) pasan a ser propiedad de quien los ha capturado . Sin embargo, si la guerra se libra por la autoridad de derecho, eso es, por defender leyes, y el príncipe tiene jurisdicción sobre sus enemigos, puede apropiarse de todos sus bienes porque se han sublevado contra su poder. Y al contrario, si el príncipe cristiano lucha contra los enemigos sobre los que no tiene jurisdicción, puede tomar de los bienes de sus adversarios solamente la cantidad que le había sido robada o destruida (pp. 466-467).

Esta última norma se extiende a cada guerra justa, porque el príncipe siempre debería luchar con modestia, y cuando invade las tierras de sus enemigos debe contentarse con la retribución por los daños producidos y "ultra aquesta quantitat dita, no deu lo just invasor prendre res si, donchs, lo colpable no merexia ésser privat de tots sos béns" (pp. 464-465). Además, la batalla justa requiere que se respeten la 
vida y los bienes de todos aquellos que no colaboran con el enemigo, aunque sean sus vasallos o súbditos. Eiximenis funda su argumento en la opinión de Hostiensis que pide una plena protección para todas las personas que no intervienen en el conflicto y al mismo tiempo acusa a los príncipes que no cumplen tal solicitud de luchar injustamente y sin legitimidad (p. 465).

El concepto de la guerra justa de Eiximenis es un concepto puramente eclesiástico en el que se sobrepone la autoridad de la Iglesia sobre el poder del mundo y se considera la fe católica como la única fe verdadera, y por consiguiente fray Francesc niega a los herejes o a los que han cometido un "leg crim" (p. 467) cualquier derecho a defender y a mantener la libertad y su propiedad. No obstante, los mandamientos divinos se aplican a todos por igual, así que incluso los cristianos que van a la guerra, aunque sea por una causa justa, tienen que hacer penitencia por sus pecados: por haber matado a sus enemigos y por haber recuperado los bienes robando. De la justicia nadie queda exento.

\section{Bibliografía}

EIXIMENIS, F. (1983), Lo Crestià, Edicions 62, Barcelona.

EIXIMENIS, F. (1986), Dotzè llibre del Crestià, vol. II.1, Collegi Universitari de Girona, Diputació de Girona, Esplugues.

GOLTZ, P. (2016), “Zagadnienia wojny i pokoju w dziele Policraticus Jana z Salisbury”, en: Kotecki, R., Maciejewski J. (ed.), Ecclesia et bellum. Kościól wobec wojny i zaangażowania militarnego duchowieństwa $w$ wiekach średnich, Wydawnictwo Uniwersytetu Kazimierza Wielkiego, Bydgoszcz, pp. 42-63.

GUIXERAS, D., MARTÍ, S. (2014), “El Crestià", en: Badia, L. (ed.), Literatura medieval (II). Segles XIV-XV, Enciclopèdia Catalana, Editorial Barcino, Barcelona, pp. 36-47.

HAUF, A. (1990), D'Eiximenis a Sor Isabel de Villena, "Biblioteca Sanchis Guarner”, 19, Institut de Filologia Valenciana, València/Barcelona.

HAUF, A. (2009), "Introducción al Regiment de la cosa pública i Dotzè del Crestià de Fray Francesc Eiximenis, OFM”, en: Eiximenis, F., Lo 
regiment de la cosa pública en el Dotzè del Crestià, Centro de Lingüística Aplicada Atenea, Madrid, pp. 11-52.

RIQUER, M. de (1986), Història de la literatura catalana, Ariel, Barcelona. 